\title{
Predição de ganho genético com diferentes índices de seleção no milho pipoca CMS-43(1)
}

\begin{abstract}
Maria José Granate(2), Cosme Damião $\operatorname{Cruz}^{(3)}$ e Cleso Antônio Patto Pacheco(4)
Resumo - O melhoramento simultâneo da capacidade de expansão e da produtividade no milho pipoca são dificultados por causa da correlação negativa entre as duas características, mas o uso de índices de seleção permite contornar essa dificuldade. Em 1997/1998 foram avaliadas 166 famílias de meiosirmãos do composto de milho pipoca (Zea mays L.) CMS-43, na Embrapa-Centro Nacional de Pesquisa de Milho e Sorgo, em Sete Lagoas, MG, no delineamento em blocos casualizados. Os índices de seleção empregados para predizer os ganhos por seleção foram os de Smith e Hazel, Pesek \& Baker, Elston e de Williams. O índice de seleção de Smith e Hazel permitiu a predição de ganhos superiores em maior número de caracteres; com o índice de seleção de Williams não se verificou nenhum dado significativo. O uso de índices de seleção é adequado porque permite a predição de ganhos simultâneos nas duas principais características.
\end{abstract}

Termos para indexação: Zea mays, características agronômicas, métodos de melhoramento.

\section{Prediction of genetic gain with different selection indexes in popcorn CMS-43}

\begin{abstract}
Simultaneous breeding for expansion volume and grain yield in popcorn (Zea mays L.) is difficult because of negative correlation between these traits. The use of selection indexes allows to overcome this difficulty. In 1997/1998, at Embrapa-Centro Nacional de Pesquisa de Milho e Sorgo, in Sete Lagoas, MG, Brazil,166 half-sib families from CMS-43 popcorn were evaluated, using random blocks design. The selection indexes used were Smith and Hazel, Pesek \& Baker, Elston and Williams. The selection index of Smith and Hazel allowed larger predicted gains in more traits, and on the contrary, the prediction using the selection index of Williams was insignificant. The use of selection indexes allows simultaneous prediction of gains in the two main traits.
\end{abstract}

Index terms: Zea mays, agronomic characters, breeding methods.

\section{Introdução}

Os índices de seleção são uma técnica multivariada que associa as informações relativas a vários caracteres de interesse agronômico com as propriedades genéticas da população avaliada. Com os ín-

(1) Aceito para publicação em 4 de setembro de 2001 .

Extraído da tese de doutorado apresentada pelo primeiro autor à Universidade Federal de Viçosa (UFV), Viçosa, MG.

(2) Empresa de Pesquisa Agropecuária de Minas Gerais, Centro Tecnológico da Zona da Mata, Vila Gianetti, 46 , Campus Universitário, CEP 36571-000 Viçosa, MG E-mail: epamig@mail.ufv.br

(3) UFV, Dep. de Biologia Geral, CEP 36571-000 Viçosa, MG E-mail: cdcruz@mail.ufv.br

(4) Embrapa-Centro Nacional de Pesquisa de Milho e Sorgo, Caixa Postal 151, CEP 35701-970 Sete Lagoas, MG. E-mail: cleso@cnpms.embrapa.br dices de seleção cria-se valor numérico, que funciona como caráter adicional, teórico, resultante da combinação de determinados caracteres escolhidos pelo melhorista, sobre os quais se deseja exercer a seleção simultânea (Cruz \& Regazzi, 1994). Os índices de seleção foram propostos por Smith (1936), e a sua eficiência na seleção simultânea de características é superior ao método tandem, em que a seleção é feita em uma característica de cada vez, e ao dos níveis independentes de eliminação, no qual se estabelece um nível, máximo ou mínimo, e a seleção é feita entre os indivíduos que se encontram entre esse nível e o nível desejado (Haarman et al., 1993).

Em milho pipoca, a correlação negativa entre as duas principais características de interesse econômico, a capacidade de expansão e a produtividade, dificulta o melhoramento mas não o impossibilita. 
Segundo Marques (2000), a predição de ganhos simultâneos nessas características não foi possível, usando o método de seleção direta e seleção indireta (resposta correlacionada), pelo que a utilização de índices de seleção é mais indicada.

Cruz et al. (1993) verificaram ganhos simultâneos nas características teor de óleo e rendimento de espigas, em progênies de irmãos completos de milho comum, usando índices de seleção, o que não foi possível quando usaram a seleção direta e indireta. Os resultados foram 3,1\% mais favoráveis com o índice base de Williams (1962) do que com o índice clássico de Smith (1936) e Hazel (1943), quando a seleção se exerceu sobre aquelas duas características. Os mesmos autores constataram ganhos maiores empregando o índice clássico quando as características principais foram rendimento de espiga, peso médio da espiga, comprimento da espiga e peso médio de 100 grãos. Nesse estudo, o índice dos ganhos desejados de Pesek \& Baker (1969) não se mostrou eficiente na predição de ganhos simultâneos, mas o índice base, o índice livre de pesos e parâmetros de Elston (1963) e o da soma de ranks foram eficientes na predição de ganhos em todos os caracteres de interesse. No melhoramento para aumento da produção de óleo de Melaleuca alternifolia (Maiden \& Betche) Cheel e de M. linariifolia Sm., na Austrália, a correlação negativa entre a produção de óleo e o peso seco da planta não permitia a predição de ganhos positivos nesses dois caracteres simultaneamente, quando se usava a predição por seleção direta e indireta. Com a utilização de índice de seleção combinada, foi possível predizer ganhos de $17 \%$ na produção de óleo e de $14 \%$ na produção de madeira, na primeira geração de seleção e com a seleção de uma árvore em dez (Butcher et al., 1996).

O índice clássico e o de Pesek \& Baker (1969) foram utilizados por Martins (1999), ao comparar métodos uni e multivariados para a seleção de famílias de meios-irmãos de Eucalyptus grandis. Concluiu que os índices foram os métodos mais eficientes, e permitiram predizer maiores ganhos e com melhor distribuição, em relação às várias características observadas. Os melhores ganhos em todas as características avaliadas foram preditos com os índices de seleção da soma de ranks, multiplicativo, livre de pesos e parâmetros e base, no melhoramento da re- sistência ao frio de duas populações de milho (Crosbie et al., 1980).

O objetivo deste trabalho foi comparar os ganhos preditos obtidos com a seleção simultânea de caracteres, utilizando vários índices de seleção e vários critérios de seleção, e escolher o índice e o critério de seleção mais adequados.

\section{Material e Métodos}

No ano agrícola de 1997/1998 foram realizados ensaios com 166 famílias de meios-irmãos do composto de milho pipoca CMS-43, na Embrapa-Centro Nacional de Pesquisa de Milho e Sorgo (CNPMS), em Sete Lagoas, MG. Este composto foi obtido pela recombinação de materiais considerados superiores e tolerantes às principais doenças existentes no Banco de Germoplasma da Embrapa-CNPMS. A partir de 1979, as populações do composto foram submetidas a mais de cinco ciclos de seleção massal estratificada e em seguida a vários ciclos de seleção entre e dentro de famílias de meios-irmãos. A seleção foi a favor de boas características agronômicas, resistência a doenças e qualidade da pipoca (Pacheco et al., 1998). As testemunhas foram a variedade híbrido comercial Zélia e a população de milho CMS-43 do ciclo anterior.

Cada parcela constou de uma fileira de $5 \mathrm{~m}$, com 50 sementes distribuídas, em 25 covas espaçadas $0,20 \mathrm{~m}$. O espaçamento entre fileiras foi de $0,90 \mathrm{~m}$. A semeadura foi efetuada manualmente e o estande ideal após o desbaste foi de 25 plantas por fileira. Foram feitas capinas com tração mecanizada quando necessário. No plantio aplicaram-se $350 \mathrm{~kg} / \mathrm{ha}$ da fórmula NPK 4-14-8, e $250 \mathrm{~kg} / \mathrm{ha}$ de sulfato de amônio, em cobertura, após o desbaste, realizado no estádio de plântula. A colheita foi feita manualmente após a maturação fisiológica.

As avaliações dos seguintes caracteres referem-se a cada parcela e foram feitas na época da colheita: ALTP: altura de planta, média das medições feitas do nível do solo à inserção da folha-bandeira, em seis plantas competitivas tomadas ao acaso, em cm; ALTES: altura da espiga, média das distâncias do nível do solo até a inserção da espiga superior, nas mesmas seis plantas, em cm; PRE: posição relativa da espiga, razão entre a altura da espiga e a altura da planta; AC: número de plantas acamadas, contagem das plantas com ângulo de inclinação superior a $45^{\circ} \mathrm{em}$ relação à vertical; QUE: número de plantas cujo colmo se apresentava quebrado; EST: estande, número de plantas; NESP: número de espigas; PROLI: prolificidade, razão entre o número de espigas e o número de plantas; PRODU: produtividade, peso dos grãos debulhados por 
unidade de área, em kg/ha; CE: capacidade de expansão, razão entre o volume de pipoca expandida e o volume de grãos antes do pipocamento. Para isso, uma amostra de $30 \mathrm{~mL}$ de grãos foi tomada de cada parcela, medida em proveta graduada, estourada em pipoqueira elétrica, regulada automaticamente para $237^{\circ} \mathrm{C}$, e o volume da pipoca expandida foi medido em proveta graduada de $1.000 \mathrm{~mL}$.

As análises de variância foram realizadas considerando o delineamento em blocos ao acaso, com duas repetições, com testemunhas adicionais. Foram avaliados 179 tratamentos, que incluíram as 166 famílias de meios-irmãos, consideradas de efeito aleatório, e 13 famílias de meiosirmãos das duas testemunhas, consideradas de efeito fixo.

A predição dos ganhos foi estimada simulando quatro ideótipos: primeiro procurando obter as famílias mais produtivas e com maior capacidade de expansão; em seguida, acrescentando-se a diminuição da altura de planta aos dois caracteres anteriores; depois o critério de seleção estabeleceu a diminuição do número de plantas quebradas, mantendo o aumento da produção e da capacidade de expansão; e por fim definiu-se o ideótipo de plantas baixas, resistentes ao acamamento, produtivas e com boa capacidade de expansão. O estimador dos ganhos preditos escolhido, para as quatro simulações, foi aquele baseado no diferencial de seleção, sendo apresentados os ganhos porcentuais. Nas quatro simulações e com os quatro índices, foi considerada a seleção das 25 melhores famílias.

Os quatro índices de seleção empregados foram o clássico de Smith (1936) e Hazel (1943), o de Williams (1962), Elston (1963) e o de Pesek \& Baker (1969). Os pesos utilizados foram o coeficiente de variação genética, o desvio-padrão genético, a razão entre o coeficiente de variação genética e o coeficiente de variação experimental, a herdabilidade, o coeficiente de correlação genética e vários pesos obtidos aleatoriamente por tentativas. A média de cada característica foi utilizada como nível de eliminação ou ponto de corte, quando se adotou o índice de Elston (1963).

O Programa Genes (Cruz, 2001) foi utilizado para realizar todas as computações.

\section{Resultados e Discussão}

O índice de seleção de Smith (1936) e Hazel (1943) não permitiu a estimação da predição de ganhos simultâneos nos caracteres PRODU e CE, quando os pesos atribuídos foram o $\mathrm{CV}_{\mathrm{g}}$, o desvio-padrão genético, a razão $\mathrm{CV}_{\mathrm{g}} / \mathrm{CV}$ e a herdabilidade (Tabela 1). Com esses quatro conjuntos de pesos, os ganhos preditos em PRODU foram iguais assim como as per- das na capacidade de expansão. Após várias tentativas, atribuindo pesos de várias grandezas aleatoriamente, conseguiu-se predizer ganhos simultâneos nas duas características principais, sendo o ganho porcentual conjunto de $12,13 \%$. O maior ganho em CE foi predito por este índice. Com os pesos obtidos por tentativas, as outras características também apresentaram ganhos preditos de interesse para este estudo. Em relação a ALTP e ALTES esses ganhos, apesar de poderem ser considerados muito pequenos, não são desejáveis. Os ganhos preditos para as características PRE, AC e QUE foram negativos, o que é interessante, e os ganhos preditos para os caracteres NESP e PROLI podem ser considerados adequados.

O coeficiente de variação genético foi considerado por Cruz (1990) como o peso mais apropriado para estimar ganhos usando índices de seleção, em milho comum. Martins (1999), em eucalipto, ao utilizar o coeficiente de variação genético como peso do índice clássico (Smith, 1936; Hazel, 1943), não obteve os resultados desejados, e então optou por usar como pesos valores baseados em estatísticas dos próprios dados.

O índice de seleção de Pesek \& Baker (1969) permitiu predizer ganhos ligeiramente superiores aos do índice de Smith (1936) e Hazel (1943) em relação a PRODU e CE, sendo o porcentual conjunto de $12,34 \%$, quando os ganhos desejados foram iguais a um desvio-padrão genético, mas não estimou perdas em ALTP e ALTES. Quando se estabeleceu o valor dos ganhos desejados como iguais a $10 \%$ da média dos caracteres, os ganhos porcentuais preditos somaram $10,45 \%$ em relação a PRODU e CE. Nas outras características os resultados fornecidos por este índice foram semelhantes aos preditos com o índice clássico. Martins (1999), em eucalipto, considerou o índice de Pesek \& Baker (1969) mais eficiente do que o índice clássico.

O índice de seleção livre de pesos e parâmetros (Elston, 1963) estimou ganhos preditos semelhantes aos outros dois índices, quando se usou a média das características como ponto de corte; o ganho porcentual conjunto para PRODU e CE foi de $11,02 \%$. Os ganhos nas outras características foram semelhantes aos dos outros índices. 
O índice de seleção base (Williams, 1962) não permitiu a obtenção de estimativas de ganhos preditos simultâneos nas duas características de interesse.

O índice de seleção de Smith (1936) e Hazel (1943) foi eficiente na predição do progresso desejado nos caracteres ALTP, PRODU e CE apenas quando se usaram pesos obtidos aleatoriamente, por tentativas (Tabela 2). Os ganhos porcentuais conjuntos, previstos para as duas características mais importantes, PRODU e CE, foram de $9,14 \%$. O ganho na característica CE, com este critério de seleção, foi superior ao estimado usando o critério de seleção anterior (plantas mais produtivas e de maior capacidade de expansão). Com a seleção nas características ALTP, PRODU e CE foi possível prever redução em ALTP, ALTES, PRE e QUE, o que é desejável. No entanto, houve previsão de aumento no número de plantas acamadas. Os outros caracteres apresentaram previsão de ganhos. Os ganhos preditos em conjunto, referente às duas principais características, com este índice, foram bastante superiores aos preditos pelos outros índices de seleção.
O índice de seleção de Pesek \& Baker (1969) estimou ganhos preditos conjuntos para PRODU e CE de 5,30\% quando se usou o desvio-padrão genético como o valor dos ganhos desejados; a seleção foi exercida para diminuição de ALTP e aumento de PRODU e CE. As outras características apresentaram ganhos preditos de pequena magnitude.

Os ganhos preditos usando o índice de seleção livre de pesos e parâmetros foram, em relação ao conjunto das características PRODU e CE, 7,13\% inferiores aos do índice clássico, mas foram superiores aos do índice de Pesek \& Baker (1969). As outras características apresentaram ganhos, com exceção de AC e EST.

O índice de seleção base não apresentou ganhos simultâneos nas duas principais características.

A predição dos ganhos por seleção para diminuição de plantas quebradas, aumento da produção e da capacidade de expansão, é apresentada na Tabela 3; a seleção foi praticada nos caracteres QUE, PRODU e CE. Os índices de seleção de Smith (1936) e Hazel (1943) apresentaram ganhos preditos, em conjunto, para as duas principais características, supe-

Tabela 1. Estimativas dos ganhos porcentuais, baseados em diferentes índices de seleção, por seleção simultânea para aumento dos caracteres PRODU e CE, avaliados em famílias de meios-irmãos do milho pipoca CMS-43, em Sete Lagoas, MG, no ano agrícola de 1997/1998, para formação de uma população com 25 famílias ${ }^{(1)}$

\begin{tabular}{|c|c|c|c|c|c|c|c|c|c|c|c|}
\hline \multirow[t]{2}{*}{ Caracteres } & \multicolumn{11}{|c|}{ Índices de seleção } \\
\hline & $\mathrm{SHCV}_{\mathrm{g}}$ & SHô ${ }_{g}$ & $\mathrm{SHCV}_{\mathrm{g}} / \mathrm{CV}_{\mathrm{e}}$ & $\mathrm{SHh}^{2}$ & $\mathrm{SHr}_{\mathrm{g}}$ & SHt1 & SHt2 & $\mathrm{PB} \hat{\sigma}_{\mathrm{g}}$ & $\mathrm{PB} 10 \% \mathrm{~m}$ & LPP & $\mathrm{BCV}_{\mathrm{g}}$ \\
\hline ALTP & 0,63 & 0,63 & 0,63 & 0,63 & 0,63 & 0,26 & 0,53 & 0,61 & 0,26 & 0,54 & 0,51 \\
\hline ALTES & 0,69 & 0,69 & 0,69 & 0,69 & 0,69 & 0,05 & 0,22 & 0,37 & 0,05 & 0,16 & 0,47 \\
\hline PRE & Ind. & Ind. & Ind. & Ind. & Ind. & $-0,01$ & $-0,02$ & $-0,02$ & $-0,01$ & $-0,02$ & Ind. \\
\hline $\mathrm{AC}$ & $-2,32$ & $-2,32$ & $-2,32$ & $-2,32$ & $-2,32$ & 0,18 & $-0,35$ & 0,22 & 0,18 & $-0,31$ & $-0,97$ \\
\hline QUE & 2,41 & 2,41 & 2,41 & 2,41 & 2,41 & $-2,71$ & $-1,81$ & $-2,01$ & $-2,71$ & $-3,02$ & 1,00 \\
\hline EST & 0,11 & 0,11 & 0,11 & 0,11 & 0,11 & 0,08 & 0,19 & 0,19 & 0,08 & 0,10 & 0,18 \\
\hline NESP & 10,08 & 10,08 & 10,08 & 10,08 & 10,08 & 4,04 & 6,98 & 8,21 & 4,04 & 6,10 & 10,96 \\
\hline PROLI & 8,46 & 8,46 & 8,46 & 8,46 & 8,46 & 3,18 & 5,28 & 6,37 & 3,18 & 5,10 & 8,83 \\
\hline PRODU & 10,65 & 10,65 & 10,65 & 10,65 & 10,65 & 4,22 & 7,50 & 8,27 & 4,22 & 6,08 & 10,93 \\
\hline $\mathrm{CE}$ & $-3,42$ & $-3,42$ & $-3,42$ & $-3,42$ & $-3,42$ & 6,53 & 4,63 & 4,07 & 6,23 & 4,94 & $-1,18$ \\
\hline
\end{tabular}

(1)SH: índice de seleção de Smith (1936) e Hazel (1943); $\mathrm{SHCV}_{\mathrm{g}}$ : SH usando o coeficiente de variação genético como peso econômico; SH $\hat{\sigma}_{\mathrm{g}}$ : SH usando o desvio-padrão genético como peso econômico; $\mathrm{SHCV}_{\mathrm{g}} / \mathrm{CV}_{\mathrm{e}}$ : $\mathrm{SH}$ usando a razão $\mathrm{CV}_{\mathrm{g}} / \mathrm{CV}_{\mathrm{e}}$ como peso econômico; $\mathrm{SH}{ }^{2}$ : $\mathrm{SH}$ usando a herdabilidade como peso econômico; $\mathrm{SHr}_{\mathrm{g}}$ : SH usando o coeficiente de correlação genético com CE como peso econômico; SHt1: SH usando pesos econômicos $1 \mathrm{e}$ 450 para PRODU e CE, obtidos por tentativas; $\mathrm{SHt} 2$ : SH usando pesos econômicos 1 e 350 para PRODU e CE, obtidos por tentativas; PB $\hat{\sigma}_{\mathrm{g}}$ : índice de seleção de Pesek \& Baker (1969) usando como ganhos desejados o desvio-padrão genético; PB10\%m: índice de seleção de Pesek \& Baker (1969) usando como ganhos desejados $10 \%$ da média dos caracteres; LPP: índice de seleção livre de pesos e parâmetros (Elston, 1963) usando a média como ponto de corte; $\mathrm{BCV}_{\mathrm{g}}$ : índice de seleção base (Williams, 1962) usando o $\mathrm{CV}_{\mathrm{g}}$ como peso econômico. Ind.: indeterminado; ALTP: altura de planta em cm; ALTES: altura de espiga em cm; PRE: posição relativa da espiga; AC: número de plantas acamadas por parcela; QUE: número de plantas quebradas por parcela; EST: número de plantas por parcela; NESP: número de espigas por parcela; PROLI: número de espigas por planta; PRODU: produtividade (peso das espigas, em $\mathrm{kg} / \mathrm{ha}$ ); CE: capacidade de expansão 
Tabela 2. Estimativas dos ganhos porcentuais, baseados em diferentes índices de seleção, por seleção simultânea para diminuição do caráter ALTP, e para aumento dos caracteres PRODU e CE, avaliados nas famílias de meios-irmãos do milho pipoca CMS-43, em Sete Lagoas, MG, no ano agrícola de 1997/1998, para formação de uma população com 25 famílias ${ }^{(1)}$.

\begin{tabular}{|c|c|c|c|c|c|c|c|c|c|c|c|}
\hline \multirow[t]{2}{*}{ Caracteres } & \multicolumn{11}{|c|}{ Índices de seleção } \\
\hline & $\mathrm{SHCV}_{\mathrm{g}}$ & $\mathrm{SH} \hat{\sigma}_{\mathrm{g}}$ & $\mathrm{SHCV}_{\mathrm{g}} / \mathrm{CV}_{\mathrm{e}}$ & $\mathrm{SHh}^{2}$ & $\mathrm{SHr}_{\mathrm{g}}$ & SHt1 & SHt2 & $\mathrm{PB} \hat{\sigma}_{\mathrm{g}}$ & PB $10 \% \mathrm{~m}$ & LPP & $\mathrm{BCV}_{\mathrm{g}}$ \\
\hline ALTP & 0,96 & 0,96 & 1,11 & 0,96 & 0,96 & $-0,39$ & $-0,15$ & $-1,59$ & $-1,77$ & $-0,90$ & 0,38 \\
\hline ALTES & 0,98 & 0,98 & 1,10 & 0,98 & 0,98 & $-0,67$ & $-0,38$ & $-1,36$ & $-1,64$ & $-0,98$ & 0,38 \\
\hline PRE & Ind. & Ind. & Ind. & Ind. & Ind. & $-0,02$ & $-0,01$ & 0,02 & 0,01 & $-0,01$ & Ind. \\
\hline $\mathrm{AC}$ & $-1,29$ & $-1,29$ & $-1,50$ & $-1,29$ & $-1,29$ & 2,02 & 1,90 & 1,12 & 1,37 & 1,41 & $-0,76$ \\
\hline QUE & 0,40 & 0,40 & 0,60 & 0,40 & 0,40 & $-1,21$ & $-2,61$ & 0,30 & 1,00 & $-4,12$ & 1,91 \\
\hline EST & 0,13 & 0,13 & 0,11 & 0,13 & 0,13 & $-0,02$ & 0,02 & 0,08 & 0,07 & $-0,04$ & 0,21 \\
\hline NESP & 9,14 & 9,14 & 9,05 & 9,14 & 9,14 & 0,46 & 2,08 & 3,99 & 2,67 & 1,59 & 11,50 \\
\hline PROLI & 7,45 & 7,45 & 7,49 & 7,45 & 7,45 & 0,60 & 1,73 & 3,29 & 2,12 & 1,86 & 9,14 \\
\hline PRODU & 10,27 & 10,27 & 10,22 & 10,27 & 10,27 & 0,88 & 2,12 & 3,22 & 1,36 & 2,84 & 10,93 \\
\hline $\mathrm{CE}$ & $-3,99$ & $-3,99$ & $-3,70$ & $-3,99$ & $-3,99$ & 7,10 & 7,02 & 2,08 & 2,57 & 4,29 & $-1,36$ \\
\hline
\end{tabular}

${ }^{(1)}$ SH: índice de seleção de Smith (1936) e Hazel (1943); SHCV $_{\mathrm{g}}$ SH usando o coeficiente de variação genético como peso econômico; SH $\hat{\sigma}_{\mathrm{g}}$ : SH usando o desvio-padrão genético como peso econômico; $\mathrm{SHCV}_{\mathrm{g}} / \mathrm{CV}_{\mathrm{e}}$ : $\mathrm{SH}$ usando a razão entre o coeficiente de variação genético e o coeficiente de variação residual como peso econômico; $\mathrm{SHh}^{2}$ : $\mathrm{SH}$ usando a herdabilidade como peso econômico; $\mathrm{SHr}_{\mathrm{g}}$ : $\mathrm{SH}$ usando o coeficiente de correlação genético com CE como peso econômico; SHt1: SH usando pesos econômicos: -60, 1 e 400 para ALT, PRODU e CE respetivamente, obtidos por tentativas; SHt2: SH usando pesos econômicos: -50, 1 e 350 para ALTP, PRODU e CE respetivamente, obtidos por tentativas; PB $\hat{\sigma}_{g}$ : índice de seleção de Pesek \& Baker (1969) usando como ganhos desejados o desvio-padrão genético; PB10\%m: índice de seleção de Pesek \& Baker (1969) usando como ganhos desejados 10\% da média dos caracteres; LPP: índice de seleção livre de pesos e parâmetros (Elston, 1963) usando como pontos de corte a média mais $\hat{\sigma}_{\mathrm{g}}$ para ALTP, a média menos $\hat{\sigma}_{\mathrm{g}}$ para PRODU e a média para $\mathrm{CE} ; \mathrm{BCV}_{\mathrm{g}}$ : índice de seleção base (Williams, 1962) usando o $\mathrm{CV}_{\mathrm{g}}$ como peso econômico; Ind.: indeterminado; ALTP: altura de planta em cm; ALTES: altura de espiga em cm; PRE: posição relativa da espiga; AC: número de plantas acamadas por parcela; QUE: número de plantas quebradas por parcela; EST: número de plantas por parcela; NESP: número de espigas por parcela; PROLI: número de espigas por planta; PRODU: produtividade (peso das espigas, em kg/ha); CE: capacidade de expansão.

Tabela 3. Estimativas dos ganhos porcentuais, baseados em diferentes índices de seleção, por seleção simultânea para diminuição do caráter QUE, e para aumento dos caracteres PRODU e CE, avaliados nas famílias de meios-irmãos do milho pipoca CMS-43, em Sete Lagoas, MG, no ano agrícola de 1997/1998, para formação de uma população com 25 famílias ${ }^{(1)}$.

\begin{tabular}{|c|c|c|c|c|c|c|c|c|c|c|c|}
\hline \multirow[t]{2}{*}{ Caracteres } & \multicolumn{11}{|c|}{ Índices de seleção } \\
\hline & $\mathrm{SHCV}_{\mathrm{g}}$ & $\mathrm{SH} \hat{\sigma}_{\mathrm{g}}$ & $\mathrm{SHCV}_{\mathrm{g}} / \mathrm{CV}_{\mathrm{e}}$ & $\mathrm{SHh}^{2}$ & $\mathrm{SHr}_{\mathrm{g}}$ & SHt1 & SHt2 & $\mathrm{PB} \hat{\sigma}_{\mathrm{g}}$ & $\mathrm{PB} 10 \% \mathrm{~m}$ & LPP & $\mathrm{BCV}_{\mathrm{g}}$ \\
\hline ALTP & 0,63 & 0,63 & 0,55 & 0,63 & 0,63 & 0,61 & 0,37 & 0,57 & 0,41 & 0,56 & 0,51 \\
\hline ALTES & 0,69 & 0,69 & 0,64 & 0,69 & 0,69 & 0,37 & 0,12 & 0,43 & 0,17 & 0,26 & 0,47 \\
\hline PRE & Ind. & Ind. & Ind. & Ind. & Ind. & $-0,02$ & $-0,02$ & $-0,01$ & $-0,02$ & $-0,02$ & Ind. \\
\hline $\mathrm{AC}$ & $-2,32$ & $-2,32$ & $-1,91$ & $-2,32$ & $-2,32$ & 0,22 & $-0,60$ & 1,20 & 1,37 & 1,94 & $-0,97$ \\
\hline QUE & 2,41 & 2,41 & 2,01 & 2,41 & 2,41 & $-2,01$ & $-2,41$ & $-7,84$ & $-7,03$ & $-9,74$ & 1,00 \\
\hline EST & 0,11 & 0,11 & 0,06 & 0,11 & 0,11 & 0,19 & 0,14 & 0,03 & $-0,06$ & Ind. & 0,18 \\
\hline NESP & 10,08 & 10,08 & 9,98 & 10,08 & 10,08 & 8,21 & 5,46 & 5,51 & 2,96 & $-0,08$ & 10,96 \\
\hline PROLI & 8,46 & 8,46 & 8,69 & 8,46 & 8,46 & 6,37 & 4,09 & 4,97 & 3,10 & $-0,13$ & 8,83 \\
\hline PRODU & 10,65 & 10,65 & 10,70 & 10,65 & 10,65 & 8,27 & 5,46 & 5,88 & 2,84 & 0,93 & 10,93 \\
\hline $\mathrm{CE}$ & $-3,43$ & $-3,43$ & $-3,26$ & $-3,43$ & $-3,43$ & 4,08 & 5,91 & 4,69 & 6,53 & 5,71 & $-1,18$ \\
\hline
\end{tabular}

${ }^{(1)} \mathrm{SH}$ : índice de seleção de Smith (1936) e Hazel (1943); $\mathrm{SHCV}_{\mathrm{g}}$ : SH usando o coeficiente de variação genético como peso econômico; SH $\hat{\sigma}_{\mathrm{g}}$ : SH usando o desvio-padrão genético como peso econômico; $\mathrm{SHCV}_{\mathrm{g}} / \mathrm{CV}_{\mathrm{e}}$ : $\mathrm{SH}$ usando a razão entre o coeficiente de variação genético e o coeficiente de variação residual como peso econômico; $\mathrm{SHh}^{2}$ : $\mathrm{SH}$ usando a herdabilidade como peso econômico; $\mathrm{SHr}$ : $\mathrm{SH}$ usando o coeficiente de correlação genético com a CE como peso econômico; SHt1: SH usando os pesos econômicos: -10, 1 e 300 para QUE, PRODU e CE respetivamente, obtidos por tentativas;

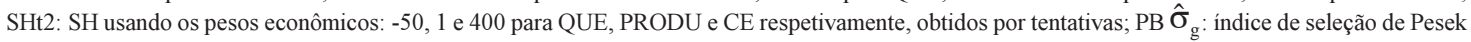
\& Baker (1969) usando como ganhos desejados o desvio-padrão genético; PB10\%m: índice de seleção de Pesek \& Baker (1969) usando como ganhos desejados $10 \%$ da média dos caracteres; LPP: índice de seleção livre de pesos e parâmetros (Elston, 1963) usando como pontos de corte a média; $\mathrm{BCV}_{\mathrm{g}}$ : índice de seleção base (Williams, 1962) usando o $\mathrm{CV}_{\mathrm{g}}$ como peso econômico; Ind.: indeterminado; ALTP: altura de planta em cm; ALTES: altura de espiga em cm; PRE: posição relativa da espiga; AC: número de plantas acamadas por parcela; QUE: número de plantas quebradas por parcela; EST: número de plantas por parcela; NESP: número de espigas por parcela; PROLI: número de espigas por planta; PRODU: produtividade (peso das espigas, em kg/ha); CE: capacidade de expansão. 
riores aos dos outros índices, quando se usaram pesos obtidos aleatoriamente por tentativas. O porcentual conjunto foi de $12,35 \%$, para as características PRODU e CE. Foi possível predizer a diminuição dos caracteres QUE e PRE, mas não se obteve a diminuição prevista com relação às características ALTP e ALTES. As outras características apresentaram aumentos.

O índice de seleção de Pesek \& Baker (1969) estimou uma diminuição na característica QUE bastante superior aos outros índices, embora o ganho conjunto nas duas características principais tenha sido inferior. As características restantes apresentaram comportamento semelhante ao apresentado com o índice de Smith (1936) e Hazel (1943).

Com o índice livre de pesos e parâmetros, o ganho predito em conjunto, para as características PRODU e CE, foi de 6,64\% e não foram estimados ganhos para a característica EST. O índice base não permitiu a predição de ganhos simultâneos em relação às características PRODU e CE e não estimou ganhos para a característica PRE.
A predição dos ganhos por seleção para diminuição da altura de planta, diminuição do número de plantas quebradas, aumento da produção e aumento da capacidade de expansão é apresentada na Tabela 4; a seleção foi praticada nos caracteres ALTP, QUE, PRODU e CE. O índice de Smith (1936) e Hazel (1943) apresentou ganhos nas duas características principais com os pesos econômicos obtidos por tentativas, designados por SHt2, com o qual o ganho predito conjunto nas duas características principais foi de $11,26 \%$. Houve aumentos nas características que se pretendia diminuir, de $0,76 \%$ na ALTP, de $0,18 \%$ na ALTES e de $0,42 \%$ no AC, o que pode ser considerado de menor importância. As famílias selecionadas por este índice são apresentadas na Tabela 5 .

O índice de Elston (1963) predisse aumentos nas duas características principais, mas acompanhados por ganhos indesejáveis em algumas das outras. O índice de Pesek \& Baker (1969) e o índice base não permitiram estimar ganhos preditos simultâneos nas duas características principais.

Tabela 4. Estimativas dos ganhos porcentuais, baseados em diferentes índices de seleção, por seleção simultânea para diminuição dos caracteres ALTP e QUE, e para aumento de PRODU e CE, avaliados nas famílias de meios-irmãos do milho pipoca CMS-43, em Sete Lagoas, MG, no ano agrícola de 1997/1998, para formação de uma população com 25 famílias ${ }^{(1)}$.

\begin{tabular}{|c|c|c|c|c|c|c|c|c|c|c|c|}
\hline \multirow{2}{*}{$\begin{array}{l}\text { Índices de } \\
\text { seleção }\end{array}$} & \multicolumn{11}{|c|}{ Seleção para diminuição de ALTP e QUE e para aumento de PRODU e CE } \\
\hline & $\mathrm{SHCV}_{\mathrm{g}}$ & SH $\hat{\sigma}_{g}$ & $\mathrm{SHCV}_{\mathrm{g}} / \mathrm{CV}_{\mathrm{e}}$ & $\mathrm{SHh}^{2}$ & $\mathrm{SHr}_{\mathrm{g}}$ & SHt1 & SHt2 & PB $\hat{\sigma}_{g}$ & PB $10 \% \mathrm{~m}$ & LPP & $\mathrm{BCV}_{\mathrm{g}}$ \\
\hline ALTP & 0,96 & 0,96 & 1,03 & 0,96 & 0,96 & $-0,18$ & 0,76 & 1,42 & 1,42 & $-0,94$ & 0,41 \\
\hline ALTES & 0,98 & 0,98 & 1,13 & 0,98 & 0,98 & $-0,45$ & 0,18 & 0,96 & 0,96 & $-0,71$ & 0,38 \\
\hline PRE & Ind. & Ind. & 0,01 & Ind. & Ind. & $-0,02$ & $-0,04$ & $-0,02$ & $-0,02$ & 0,01 & Ind. \\
\hline $\mathrm{AC}$ & $-1,29$ & $-1,29$ & $-1,33$ & $-1,29$ & $-1,29$ & $-1,95$ & 0,42 & $-3,05$ & $-3,05$ & 2,02 & $-0,93$ \\
\hline QUE & 0,40 & 0,40 & $-0,40$ & 0,40 & 0,40 & 11,05 & $-1,61$ & 13,16 & 13,16 & $-7,64$ & 1,10 \\
\hline EST & 0,13 & 0,13 & 0,13 & 0,13 & 0,13 & $-0,04$ & 0,09 & $-0,14$ & $-0,14$ & 0,06 & 0,19 \\
\hline NESP & 9,14 & 9,14 & 9,19 & 9,14 & 9,14 & 2,47 & 6,00 & 0,02 & 0,02 & $-0,18$ & 10,96 \\
\hline PROLI & 7,45 & 7,45 & 7,50 & 7,45 & 7,45 & 2,39 & 4,95 & 0,71 & 0,71 & $-0,29$ & 8,78 \\
\hline PRODU & 10,27 & 10,27 & 10,27 & 10,27 & 10,27 & 1,17 & 5,65 & $-1,12$ & $-1,12$ & 1,98 & 10,93 \\
\hline $\mathrm{CE}$ & $-3,99$ & $-3,99$ & $-3,72$ & $-3,99$ & $-3,99$ & 4,67 & 5,61 & 0,28 & 0,28 & 3,13 & $-1,19$ \\
\hline
\end{tabular}

${ }^{(1)} \mathrm{SH}$ : índice de seleção de Smith (1936) e Hazel (1943); $\mathrm{SHCV}_{\mathrm{g}}$ : SH usando o coeficiente de variação genético como peso econômico; SH $\hat{\sigma}_{\mathrm{g}}$ : SH usando o desvio-padrão genético como peso econômico; $\mathrm{SHCV}_{\mathrm{g}} / \mathrm{CV}_{\mathrm{e}}$ : $\mathrm{SH}$ usando a razão entre o coeficiente de variação genético e coeficiente de variação residual como peso econômico; $\mathrm{SHh}^{2}$ : $\mathrm{SH}$ usando a herdabilidade como peso econômico; $\mathrm{SHr}$ : $\mathrm{SH}$ usando o coeficiente de correlação genético com a CE como peso econômico; SHt1: SH usando pesos econômicos: -60, -10, 1 e 300 para ALT, QUE, PRODU e CE respetivamente, obtidos por tentativas; SHt2: SH usando pesos econômicos: -10, -10, 1 e 350 para ALTP, QUE, PRODU e CE respetivamente, obtidos por tentativas; PB $\hat{\sigma}_{\mathrm{g}}$ : índice de seleção de Pesek \& Baker (1969) usando como ganhos desejados o desvio-padrão genético; PB10\%m: índice de seleção de Pesek \& Baker (1969) usando como ganhos desejados $10 \%$ da média dos caracteres; LPP: índice de seleção livre de pesos e parâmetros (Elston, 1963) usando como pontos de corte a média mais $\hat{\sigma}_{\mathrm{g}}$, para ALTP e QUE e a média menos $\hat{\sigma}_{\mathrm{g}}$ para PRODU e CE; BCV : índice de seleção base (Williams, 1962) usando o CV $\mathrm{g}$ como peso econômico; Ind.: indeterminado; ALTP: altura de planta em cm; ALTES: altura de espiga em cm; PRE: posição relativa da espiga; AC: número de plantas acamadas por parcela; QUE: número de plantas quebradas por parcela; EST: número de plantas por parcela; NESP: número de espigas por parcela; PROLI: número de espigas por planta; PRODU: produtividade (peso das espigas, em kg/ha); CE: capacidade de expansão. 
Tabela 5. Vinte e cinco famílias selecionadas, usando diferentes índices e critérios de seleção, sendo a seleção praticada em diversos caracteres, avaliados em famílias de meios-irmãos do milho pipoca CMS-43, em Sete Lagoas, MG, no ano agrícola de 1997/1998 (1).

\begin{tabular}{|c|c|}
\hline $\begin{array}{l}\text { Índices } \\
\text { de seleção }\end{array}$ & Famílias de meios-irmãos \\
\hline & Selecionadas em relação aos caracteres PRODU e CE \\
\hline SHt2a & $1,12,13,17,20,21,23,27$ \\
\hline & \\
\hline & $8,11,12,13,17,20,24,27,30,35,48,68,85,99,10$ \\
\hline $\mathrm{SHt} 2 \mathrm{~b}$ & $\begin{array}{c}\text { Selecionadas em relação aos caracteres ALTP, PRODU e CE } \\
6,8,11,13,17,18,27,28,42,56,57,68,85,98,104,113,116,120,126,132,144,146,150,158,162\end{array}$ \\
\hline PB $\hat{\sigma}_{g}$ a & $2,5,48,53,56,58,59,65,68,69,79,85,89,90,98,100,113,114,120,127,132,153,158,162,166$ \\
\hline $\mathrm{LPPb}$ & $2,5,28,48,53,54,56,68,74,9,85,94,99,104,113,116,120,122,126,136,146,154,158,162,166$ \\
\hline & $\begin{array}{r}\text { Selecionadas em relaçã } \\
8,11,12,13,17,20,21,23,27,30,53,68,85,1\end{array}$ \\
\hline PB $\hat{\sigma}_{g} b$ & $6,8,12,13,17,20,27,30,50,54,68,94,99,104,111$, \\
\hline LPPc & $6,8,12,13,17,18,27,30,34,36,40,42,54,94,104,113,115,116,120,126,137,147,150,158,164$ \\
\hline It $2 \mathrm{c}$ & $\begin{array}{c}\text { Selecionadas em relação aos caracteres ALTP, QUE, PRODU e CE } \\
8,11,12,13,17,18,20,21,23,24,27,35,68,85,104,113,116,120,126,132,144,146,148,158\end{array}$ \\
\hline \multicolumn{2}{|c|}{$\begin{array}{l}\text { (1)SH: índice de seleção de Smith (1936) e Hazel (1943); SHt1: SH usando os pesos } 10,1 \text { e } 300 \text { para QUE, PRODU e CE, respectivamente, obtidos por } \\
\text { tentativas; SHt2a: SH usando pesos } 1 \text { e } 350 \text { para PRODU e CE, obtidos por tentativas; SHt2b: SH usando pesos } 50,1 \text { e } 350 \text { para ALTP, PRODU e } \\
\text { CE, respectivamente, obtidos por tentativas; SHt2c: SH usando os pesos -10, -10, } 1 \text { e } 350 \text { para ALTP, QUE, PRODU e CE, respectivamente, obtidos } \\
\text { por tentativas; PB10\%m: índice de seleção de Pesek \& Baker (1969) usando como ganhos desejados 10\% da média dos caracteres; PB } \hat{\sigma}_{\mathrm{g}} \text { a: índice de } \\
\text { seleção de Pesek \& Baker (1969) usando como ganhos desejados o desvio-padrão genético; PB } \hat{\sigma}_{\mathrm{g}} \text { b: indice de seleção de Pesek \& Baker (1969) usando } \\
\text { como ganhos desejados o desvio-padrão genético; LPPa: indice de seleção livre de pesos e parâmetros (Elston, 1963) usando a média como ponto de } \\
\text { corte, para PRODU e CE; LPPb: índice de seleção livre de pesos e parâmetros (Elston, 1963) usando como pontos de corte a média mais } \hat{\sigma}_{\mathrm{g}} \text { para ALTP, } \\
\text { a média menos } \hat{\sigma}_{\mathrm{g}} \text { para PRODU e a média para CE; LPPc: indice de seleção livre de pesos e parâmetros (Elston, 1963) usando a média como ponto de } \\
\text { corte para QUE, PRODU e CE; ALTP: altura de planta em cm; QUE: número de plantas quebradas por parcela; PRODU: peso das espigas, em kg/ha; } \\
\text { CE: capacidade de expansão. }\end{array}$} \\
\hline
\end{tabular}

Assim, a seleção praticada simultaneamente nas quatro características ALTP, QUE, PRODU e CE não forneceu previsão de ganhos satisfatórios com nenhum dos índices.

$\mathrm{Na}$ Tabela 5 apresentam-se as 25 famílias selecionadas usando os três índices que permitiram melhores ganhos preditos conjuntos para PRODU e $\mathrm{CE}$; a seleção foi praticada nesses caracteres. Existem 18 famílias selecionadas em comum pelos três índices. Também apresentam-se as 25 famílias selecionadas usando os três índices que permitiram maiores ganhos porcentuais conjuntos em relação aos caracteres PRODU e CE; a seleção foi exercida sobre as características ALTP, PRODU e CE. Neste caso, entre as 25 famílias selecionadas por índice, sete foram selecionadas em comum pelos três índices utilizados. São apresentadas ainda as 25 famílias selecionadas, por seleção simultânea nas características QUE, PRODU e CE, que revelaram maior porcentual conjunto de ganhos preditos para os caracteres PRODU e CE (Tabela 5). Verifica-se que existem 12 famílias comuns, selecionadas pelos três índices apresentados.

As famílias 8, 12, 13, 17, 27, 68, 104, 113, 120, 126, 147,158 e 162 foram as selecionadas mais vezes, portanto são indicadas para serem recombinadas e darem origem ao próximo ciclo de seleção.

A utilização do índice de seleção de Smith (1936) e Hazel (1943) é um procedimento viável no melhoramento simultâneo de vários caracteres, nesta população, podendo atender a diversos critérios de seleção, conforme os objetivos do melhorista.

\section{Conclusões}

1. Os ganhos preditos com o índice de seleção de Smith e Hazel são superiores aos preditos com os outros índices e manifestam-se em mais caracteres.

2. O índice de seleção de Smith e Hazel, quando utilizado para a seleção simultânea nas característi- 
cas altura de planta, produtividade e capacidade de expansão (ideótipo de plantas mais baixas, mais produtivas e de maior capacidade de expansão), com os pesos obtidos por tentativas, permite estimar os ganhos preditos considerados de mais interesse para o melhoramento desta população de milho pipoca.

3. O índice de seleção base não fornece resultados adequados.

4. O uso de índices de seleção permite a predição de ganhos simultâneos nas duas principais características (produção de grãos e capacidade de expansão).

\section{Referências}

BUTCHER, P. A.; MATHESON, A. C.; SLEE, M. U. Potential for genetic improvement of oil production in Melaleuca alterifolia and M. linariifolia. New Forest, Dordrecht, v. 11, n. 1, p. 31-51, 1996.

CROSBIE, T. M.; MOCK, J. J.; SMITH, O. S. Comparison of gains predicted by several methods for cold tolerance traits of two maize populations. Crop Science, Madison, v. 20, n. 5, p. 649-655, 1980.

CRUZ, C. D. Aplicação de algumas técnicas multivariadas no melhoramento de plantas. 1990. 188 f. Tese (Doutorado) - Escola Superior de Agricultura Luiz de Queiroz, Piracicaba.

CRUZ, C. D. Programa Genes: aplicativo computacional em genética e estatística: versão Windows. Viçosa: UFV, 2001. $648 \mathrm{p}$.

CRUZ, C. D.; REGAZZI, A. J. Modelos biométricos aplicados ao melhoramento genético. Viçosa, MG: Imprensa Universitária, 1994. 390 p.

CRUZ, C. D.; VENCOVSKY, R.; SILVA, S. O. e; TOSELLO, G. A. Comparison of gains from selection among corn progenies, based on different criteria. Revista Brasileira de Genética, Ribeirão Preto, v. 16, n. 1, p. 7989, 1993.

ELSTON, R. C. A weight-free index for the purpose of ranking or selection with respect to several traits at a time. Biometrics, Alexandria, v. 19, n. 1, p. 85-97, 1963.

HAARMAN, R. J.; WHITE, D. G.; DUDLEY, J. W. Index vs. tandem selection for improvement of grain yield, leaf blight and stalk rot resistance in maize. Maydica, Bergamo, v. 38, n. 3, p. 183-188, 1993.

HAZEL, H. N. The genetic basis for constructing selection indexes. Genetics, Menasha, v. 28, n. 6, p. 476-490, 1943.

MARQUES, M. J. B. S. G. S. M. Número mínimo de famílias de meios-irmãos de milho pipoca: critério de seleção e predição de ganhos por seleção. 2000. $236 \mathrm{f}$. Tese (Doutorado) - Universidade Federal de Viçosa, Viçosa.

MARTINS, I. S. Comparação entre métodos uni e multivariados aplicados na seleção em Eucalyptus grandis. 1999. 94 f. Tese (Doutorado) - Universidade Federal de Viçosa, Viçosa.

PACHECO, C. A. P.; GAMA, E. E. G.; GUIMARÃES, P. E. A.; SANTOS, M. X.; FERREIRA, A. S. Estimativas de parâmetros genéticos nas populações CMS-42 e CMS-43 de milho de pipoca. Pesquisa Agropecuária Brasileira, Brasília, v. 33, n. 12, p. 1995-2001, dez. 1998.

PESEK, J.; BAKER, R. J. Desired improvement in relation to selected indices. Canadian Journal of Plant Sciences, Ottawa, v. 49, n. 6, p. 803-804, 1969.

SMITH, H. F. A discriminant function for plant selection. Annals of Eugenics, London, v. 7, p. 240-250, 1936.

WILLIAMS, J. S. The evolution of a selection index. Biometrics, Alexandria, v. 18, n. 4, p. 375-393, 1962. 